\title{
Propuesta de factor de corrección a las mediciones de hemoglobina por pisos altitudinales en menores de 6 a 59 meses de edad, en el Perú Proposal of a correction factor for measurements of hemoglobin by altitudinal tiers in 6-19 month old infants in Peru
}

\author{
Marco Bartolo-Marchena ${ }^{1, a}$, Jaime Pajuelo-Ramírez ${ }^{2, a}$, Cristian Obregón-Cahuaya ${ }^{3, b}$, Catherine Bonilla- \\ Untiveros $^{4, c}$, Elizabeth Racacha-Valladares ${ }^{5, c}$, Fernando Bravo-Rebatta ${ }^{6, c}$ \\ ${ }^{1}$ Centro Nacional de Salud Intercultural, Instituto Nacional de Salud. Lima, Perú. \\ ${ }^{2}$ Instituto de Investigaciones Clínicas, Facultad de Medicina, Universidad Nacional Mayor de San Marcos. Lima, Perú. \\ ${ }^{3}$ Centro Nacional de Salud Pública, Instituto Nacional de Salud. Lima, Perú. \\ ${ }^{4}$ Universidad Científica del Sur. Lima, Perú. \\ ${ }^{5}$ Oficina General de Investigación y Transferencia Tecnológica, Instituto Nacional de Salud. Lima, Perú. \\ ${ }^{6}$ Centro Nacional de Alimentación y Nutrición, Instituto Nacional de Salud y de la Universidad Católica Sedes Sapientiae. Lima, Perú. \\ ${ }^{a}$ Médico; ${ }^{b}$ Estadistico; ${ }^{\mathrm{c}}$ Nutricionista
}

Correspondencia:

Marco A. Bartolo-Marchena marcobart@hotmail.com

Dirección: Piso 1 Urb. Vecinal Cruz de Yerbateros Mz. F Lt. 10

Distrito San Luis, Provincia Lima, Departamento Lima.

Teléfono: (511) 952024751.

Recibido: 31 octubre 2016

Aceptado: 22 mayo 2017

Conflictos de interés: Ninguno Fuente de financiamiento: Instituto Nacional de Salud

Citar como: Bartolo Marchena M, Pajuelo Ramírez J, Obregón Cahuaya C, Bonilla Untiveros C, Racacha Valladares E, Bravo Rebatta F. Propuesta de factor de corrección a las mediciones de hemoglobina por pisos altitudinales en menores de 6 a 59 meses de edad en el Perú. An Fac med. 2017;78(3): 281-286 DOI: $h$ ttp://dx.doi.org/10.15381/anales.v78i3.13759

\section{An Fac med. 2017;78(3):281-286/ http://dx.doi.org/10.15381/anales.v78i3.13759}

\section{Resumen}

Introducción. La anemia nutricional es el problema de mayor magnitud en el Perú. Objetivo. Determinar el comportamiento de la hemoglobina según pisos altitudinales, en niños peruanos, con la finalidad de proponer un factor de corrección nacional. Diseño. Descriptivo, transversal. Lugar. A nivel nacional. Participantes. 22 500 niños de 6 a 59 meses de edad. Intervenciones. Se ha usado la data de la Encuesta Demográfica y de Salud Familiar (ENDES 2015) eligiéndose, para la formulación de la propuesta, a los niños no anémicos. Se trabajó con un modelo de regresión exponencial, siendo el factor de corrección de hemoglobina por altitud: $8,3^{*} \mathrm{e}^{\left(0,000426^{*} \text { altura }\right)}-12$. Principales medidas de resultados. Prevalencia de anemia por altitud. Resultados. Se comparó la prevalencia de anémicos diagnosticados con el factor de corrección propuesto frente al usado tradicionalmente del Centers for Disease Control and Prevention (CDC). Las diferencias a nivel nacional alcanzaron al 2,5\%, evidenciándose más marcadas por encima de $3000 \mathrm{msnm}$, con 9,2\%. Los departamentos con diferencias de 5 puntos porcentuales y más fueron Junín (5,6\%), Cusco (5,7\%), Ayacucho $(6 \%)$, Pasco $(7,4 \%)$, Apurimac $(7,8 \%)$, Huancavelica $(9,9 \%)$ y Puno $(12,7 \%)$. Conclusiones. El factor de corrección propuesto identifica menor prevalencia de anemia que el factor tradicionalmente utilizado, y esta se sustenta en las mayores diferencias que se dan a partir de los $3000 \mathrm{msnm}$.

Palabras clave: Hemoglobina; Altitud; Niños; Anemia.

Abstract

Introduction: Anemia is the largest nutritional problem in Peru. Objective: To determine changes in hemoglobin level according to altitude in Peruvian children in order to propose a national correction factor. Design: Descriptive, cross-sectional study. Setting: At national level. Participants: 22500 children aged 6 to 59 months-old. Interventions: Data from the Demography and Family Health Survey (ENDES 2015) was used to select non-anemic children for the proposed formulation. An exponential regression model were used and the correction factor for hemoglobin (according to altitude) was: $8.3^{*} e^{\left(0,0004266^{* h e i g h t)}\right.}-12$. Main outcome measure: Prevalence of anemia according to altitude. Results: We compared the prevalence of anemia by calculating the hemoglobin level with the traditional formula used by the Centers for Disease Control and Prevention $(C D C)$ and with the proposed correction factor. The difference at national level reached $2.5 \%$, being greater at 3000 meters over sea level and above (9.2\%). Regions with a difference over 5 percentage points were Junín (5,6\%), Cusco (5,7\%), Ayacucho (6\%), Pasco (7,4\%), Apurímac (7,8\%), Huancavelica $(9,9 \%)$, and Puno $(12,7 \%)$. Conclusion: The proposed correction factor identifies lower prevalence of anemia compared to the traditional factor. This observation may be due to the differences found above 3000 meters over the sea level. Keywords: Hemoglobin; Altitude; Children; Anemia. 


\section{INTRODUCCIÓN}

La anemia nutricional es reconocida como el mayor problema nutricional tanto a nivel local, regional y mundial. A nivel mundial se dice que $43 \%$ de niños preescolares, 38\% de mujeres embarazadas y $29 \%$ de mujeres no embarazadas se encuentran afectadas con este problema ${ }^{(1)}$.

En el Perú existen indicios que muestran su presencia desde la época preincaica y por efecto de la colonización española. Los hallazgos del bioarqueólogo estadounidense Haagen D. Klaus en el distrito de Mórrope mostró que los esqueletos de los niños mochica presentaban lesiones en el cráneo que reflejaban parasitismo y anemia por deficiencia de hierro ${ }^{(2)}$. Estas lesiones conocidas como cribra orbitaria e hiperostosis porótica eran signos evidentes de la presencia de anemia ferropénica ${ }^{(3)}$. La presencia de hiperostosis porótica también ha sido encontrada en adultos por los investigadores Castro de la Mata ${ }^{(4)}$ y Costa-Junqueira (5). Estos hallazgos permitieron concluir que el problema de la anemia se encuentra presente desde épocas remotas.

Más recientemente se pudo observar que la preocupación de los investigadores tuvo dos etapas definidas: la primera en la que los estudios estuvieron dirigidos a conocer los mecanismos de adaptación del poblador peruano a diferentes altitudes sobre el nivel del mar; y la segunda a estudiar un problema nutricional reconocido como de Salud Pública, causada por la deficiencia de micronutrientes, específicamente del hierro, dentro de un contexto social donde la mayoría de necesidades básicas es insatisfecha.

En 1889, el médico francés Francois Gilbert Viault ascendió hasta Morococha (4 540 msnm), observando que el número de hematíes en la sangre se incrementaba entre los que ascendían, mientras que los residentes ya lo tenían elevado, lo que consideró como un fenómeno compensatorio para la vida en las grandes alturas ${ }^{(6)}$.

Posteriormente, Hurtado empezó estudios en la altura, siendo su principal preocupación observar las variaciones individuales como respuesta a la anoxemia, en función del tiempo, mencionando que los niveles de policitemia estaban relacionados al grado, duración y continuidad de la anoxemia. Así determinó que si el período de cambio era corto las variaciones de la hemoglobina $(\mathrm{Hb})$ prácticamente eran imperceptibles, encontrándose alrededor de $16 \mathrm{~g} / 100 \mathrm{~mL}$ (el estudio fue hecho en sujetos adultos que viajaron de Lima a Matucana, otros a San Mateo, Casapalca y La Cima). Diferentes fueron sus hallazgos cuando la anoxemia fue permanente; la $\mathrm{Hb}$ fue $18,8 \mathrm{~g} / 100 \mathrm{~mL}$ en La Oroya y 20,8 g/100 mL en Morococha ${ }^{(7)}$. Estos hallazgos permitieron reconocer el hecho de que a mayor altitud hay un incremento de la hipoxia y como respuesta adaptativa se observa un aumento de los glóbulos rojos y por ende de la hemoglobina, con la finalidad de poder llevar más oxígeno a los diferentes tejidos.

En el marco de la segunda etapa, los estudios tomaron otra direccionalidad. El objetivo estuvo encuadrado en conocer la magnitud y localización de los grandes problemas nutricionales y dentro de ellos la anemia nutricional, que se diagnostica por intermedio de valores de $\mathrm{Hb}$ por debajo de lo considerado 'normal', de acuerdo a lo definido por la World Health Organization (WHO) ( $\mathrm{Hb}$ por debajo de $110 \mathrm{mg} / \mathrm{L} \mathrm{u} 11 \mathrm{~g} / \mathrm{L})^{(8)}$.

En ese sentido, la primera encuesta a nivel nacional, en menores de 5 años, fue realizada por el Instituto de Nutrición (actual Centro Nacional de Alimentación y Nutrición), en el año 1975, siendo sus principales resultados que la prevalencia a nivel nacional alcanzó el 42,2\%, que en la selva se encontraban los más afectados con $57,2 \%$ y que esta prevalencia disminuía conforme se incrementaba la edad ${ }^{(9)}$. Posteriormente, a partir del año de 1996, se retomaron los estudios, cuya responsabilidad recayó en el Ministerio de Salud y en el Instituto Nacional de Estadística e Informática (INEI). El último estudio encontró una prevalencia nacional de 32,6\% (Encuesta Demográfica y de Salud Familiar-ENDES, 2015) ${ }^{(10)}$.

Dado que nuestro país es atravesado por la cordillera de los Andes, obliga a que la población tenga que vivir en diferentes altitudes y, conociendo que el comportamiento de la $\mathrm{Hb}$ es del tipo adaptativo, fue necesario utilizar factores de corrección en función de los pisos de altitud. Las encuestas realizadas en el Perú utilizaron los de Catwright ${ }^{(11)}$ y en mayor medida los del Center of Disease Control and Prevention (CDC) ${ }^{(12)}$. El más usado en la actualidad es el factor de corrección propuesto por el CDC, que se caracteriza por ser un estudio multivariado en base a la data recolectada del Pediatric Nutrition Surveillance System de niños comprendidos entre los 24 a 60 meses de edad, en ciudades representativas de EE UU, que van de 1200 a 3000 msnm y en base a una ecuación cuadrática ${ }^{(12)}$.

Las encuestas realizadas en el Perú lo han sido con niños de 6 a 59 meses y que viven hasta por encima de los 4000 msnm. Dado que el estudio del CDC no contempla a los niños menores de 24 meses ni los que viven por encima de los 3000 msnm, es necesario explorar el comportamiento de la $\mathrm{Hb}$ según pisos altitudinales, utilizando datos de niños peruanos, que cubran de alguna manera esas limitaciones metodológicas y que permitan elaborar una propuesta de corrección de $\mathrm{Hb}$ por altitud.

\section{METODOLOGÍA}

El estudio fue de tipo descriptivo, transversal. Con la finalidad de elaborar la propuesta del factor de corrección de la $\mathrm{Hb}$ por niveles de altitud, se ha elegido la información proporcionada por la ENDES 2015, y como sujeto de análisis a los niños entre 6 a 59 meses que no fuesen anémicos Esta consideración fue en base a que los identificados como anémicos no necesariamente son por déficit de hierro sino también por otras circunstancias, como déficit de vitamina B12, ácido fólico, parasitosis, infecciones, entre otros.

Bajo el supuesto que solo la altura es el factor que influye en los valores de la $\mathrm{Hb}$, el factor de corrección es una estimación de la mínima diferencia entre los niveles de $\mathrm{Hb}$ observada y la $\mathrm{Hb}$ en condiciones normales o $\mathrm{Hb}$ igual a 110 miligramos por litro. Se busca estimar la función que represente estos valores en la muestra ENDES, siguiendo los siguientes pasos: 
- Eliminar observaciones discordantes y valores extremos que influyan en el modelo (que cumplan los supuestos de significancia estadística).

- Crear la variable no observable (latente) que represente el cambio de la hemoglobina observada en altura y el valor de hemoglobina bajo condiciones normales (alturas menores de los mil metros sobre el nivel del mar) o hemoglobina igual a 110 miligramos por litro:

$Y=H b$ observada en altura -110 miligramos por litro

- Construir un modelo de regresión que determine el valor esperado la variable "y" según la función de distribución no lineal de la variable.

- Construir el intervalo de predicción (IP) para observaciones futuras (niveles de $\mathrm{Hb}$ ) según el modelo de regresión elegido.

Por tanto, según lo enunciado, el modelo a utilizar será:

$$
\hat{y} \text { - Limite } \text { inferior }_{I P}(\hat{y})
$$

En función de lo mencionado, el factor de corrección propuesto en base a un modelo de regresión exponencial es el siguiente:

Factor de corrección $=8,3 * e^{(0,000426 * \text { altura })}-12$

El límite inferior del intervalo de predicción será la curva que define cada una de las observaciones de hemoglobina bajo las condiciones:

- Valores de hemoglobina por debajo de la curva son considerados como valores anémicos.

- Valores de hemoglobina que se encuentren dentro de la curva y aquellos que se encuentren por encima son considerados como valores no anémicos.

\section{RESULTADOS}

En la tabla 1 se muestra el número de niños, así como también la prevalencia de los niños que presentaron anemia nutricional de acuerdo al factor de corrección dado por el CDC y por la propuesta del presente estudio; asimismo, la diferencia de estas prevalencias, de acuerdo a niveles de altitud.

Se observa que la prevalencia nacional dada por el factor de corrección del CDC fue $34,3 \%$ de anémicos mientras que uti-

lizando la propuesta alcanza a 31,8\%, con una diferencia de 2,5\%. Asimismo, las prevalencias van aumentando conforme lo hace la altitud, para ambas correcciones, siendo más marcadas a partir de los $3000 \mathrm{msnm}$.

Tabla 1. Número y prevalencia de anémicos, con sus diferencias, de acuerdo a factores de corrección por niveles de altitud (msnm).

\begin{tabular}{ccccc} 
& \multicolumn{4}{c}{ Prevalencia de anémicos } \\
msnm & Población & Propuesta & CDC & Diferencias \\
$\leq 500$ & 11550 & 33 & 33 & 0 \\
$501-1000$ & 1875 & 26,2 & 27,0 & $-0,8$ \\
$1001-1500$ & 970 & 25,4 & 25,7 & $-0,3$ \\
$1501-2000$ & 620 & 23,4 & 24,0 & $-0,6$ \\
$2001-2500$ & 1254 & 26,0 & 28,5 & $-2,5$ \\
$2501-3000$ & 1476 & 26,0 & 29,9 & $-3,9$ \\
$3001-3500$ & 2352 & 31,6 & 39,4 & $-7,8$ \\
$3501-4000$ & 1908 & 42,0 & 51,5 & $-9,5$ \\
$>4000$ & 563 & 43,0 & 56,8 & $-13,9$ \\
Total & 22568 & 31,8 & 34,3 & $-2,5$ \\
\hline
\end{tabular}

La tabla 2 muestra lo mismo que la anterior, pero con la diferencia de que se ha estratificado los niveles de altitud en dos: por debajo y encima de 3000 msnm. Se observa que las mayores prevalencias fueron obtenidas cuando se utilizó el factor de corrección del CDC, para ambos

niveles. Los niños que viven por encima de los 3000 msnm presentaron mayores prevalencias que su contraparte. Las diferencias de prevalencias mostradas por el mismo nivel señalan que a mayor altitud esta diferencia cuadriplica la diferencia nacional.

Tabla 2. Número y prevalencia de anémicos, con sus diferencias, de acuerdo a factores de corrección por niveles de altitud (msnm)

\begin{tabular}{ccccc} 
msnm & \multicolumn{4}{c}{ Prevalencia de anémicos } \\
$<3000$ & 17741 & 30,4 & 31,0 & $-0,6$ \\
$\geq 3000$ & 4827 & 37,0 & 46,2 & $-9,2$ \\
Total & 22568 & 31,8 & 34,3 & $-2,5$ \\
\hline
\end{tabular}

En la tabla 3 se observa la prevalencia de anemia de acuerdo a grupos de edad. La población se ha dividido en menores de 24 meses y de 24 meses a más. Las prevalencias para ambos factores de corrección fueron mayores en el grupo menor de 24 meses. Este hecho convalida la tendencia de la anemia que, conforme

se incrementa la edad, la prevalencia recorre un camino inverso. Las diferencias de las prevalencias entre ambos factores de corrección fueron similares para los grupos de edad en relación a lo hallado a nivel nacional (-2,5\%). Aplicando el factor de corrección del CDC, se muestran mayores prevalencias.

Tabla 3. Número y prevalencia de anémicos, con sus diferencias, de acuerdo a factores de corrección por grupos de edad en meses.

\begin{tabular}{ccccc} 
& \multicolumn{4}{c}{ Prevalencia de anémicos } \\
Edad & Población & Propuesta & CDC & Diferencias \\
\hline$<24$ meses & 7287 & 53,4 & 56,0 & $-2,6$ \\
$\geq 24$ meses & 15281 & 21,5 & 23,9 & $-2,4$ \\
Total & 22568 & 31,8 & 34,3 & $-2,5$ \\
\hline
\end{tabular}


La tabla 4 permite observar las diferencias que existen entre las prevalencias de anemia de acuerdo a los factores de corrección, según departamentos, incluyendo el Callao. Trece departamentos tu-

vieron prevalencias que difieren en $\leq 2 \%$; en 6 de ellos no hubo diferencias, en 4 entre 2 y $4 \%$ y los demás por encima del $5 \%$, destacando nítidamente Huancavelica con $10 \%$ y Puno con $13 \%$.

Tabla 4. Prevalencia de anémicos por departamentos, de acuerdo a factores de corrección.

\begin{tabular}{|c|c|c|c|}
\hline \multicolumn{4}{|c|}{ Prevalencia de anémicos } \\
\hline Departamento & Propuesta & $\mathrm{CDC}$ & Diferencias \\
\hline Callao & 27,4 & 27,4 & 0 \\
\hline Madre de Dios & 43,4 & 43,4 & 0 \\
\hline Lambayeque & 22,8 & 22,8 & 0 \\
\hline Ica & 32,6 & 32,6 & 0 \\
\hline Tumbes & 32,5 & 36,5 & 0 \\
\hline Ucayali & 42,7 & 42,7 & 0 \\
\hline Lima & 28,7 & 28,8 & 0,1 \\
\hline Loreto & 42,1 & 42,2 & 0,1 \\
\hline Moquegua & 27,7 & 28,4 & 0,7 \\
\hline San Martín & 29,7 & 30,4 & 0,7 \\
\hline Amazonas & 31,8 & 32,7 & 0,9 \\
\hline La Libertad & 23 & 24,2 & 1,2 \\
\hline Tacna & 25 & 26,2 & 1,2 \\
\hline Cajamarca & 22,7 & 25,3 & 2,6 \\
\hline Huánuco & 30,9 & 33,6 & 2,7 \\
\hline Arequipa & 26,3 & 29,1 & 2,8 \\
\hline Ancash & 32,7 & 36,3 & 3,6 \\
\hline Junín & 32,6 & 38,2 & 5,6 \\
\hline Cusco & 34,7 & 40,4 & 5,7 \\
\hline Ayacucho & 28,5 & 34,5 & 6 \\
\hline Pasco & 37,6 & 45 & 7,4 \\
\hline Apurímac & 33,5 & 41,3 & 7,8 \\
\hline Huancavelica & 31,9 & 41,8 & 9,9 \\
\hline Puno & 46,1 & 58,8 & 12,7 \\
\hline Total & 31,8 & 34,3 & 2,5 \\
\hline
\end{tabular}

\section{DISCUSIÓN}

En el Perú, los estudios realizados a nivel nacional para conocer la magnitud y localización de la anemia nutricional han sido por el Ministerio de Salud-por intermedio del ex Instituto de Nutrición, actualmente el Centro Nacional de Alimentación y Nutrición- y por el Instituto Nacional de Estadística e Informática (INEI), Encuestas de Demografía y de Salud Familiar (ENDES). A excepción del primer estudio realizado por el ex-Instituto de Nutrición (9), donde la toma de la muestra se hizo por venopuntura y con el factor de corrección de Catwright ${ }^{(11)}$, todos los demás, que empezaron en la década del 90, tuvieron como común denominador que la toma se hizo en sangre capilar con el
Hemo-Cue, con los mismos criterios diagnósticos y el mismo factor de corrección dado por el CDC.

La geografía de muchos países ha obligado a los investigadores estudiar el comportamiento de la $\mathrm{Hb}$ en función de la altitud. En el Perú son de destacar los estudios de Cosio ${ }^{(13)}$ y Rodriguez ${ }^{(14)}$ que mostraron, mediante el uso de fórmulas matemáticas, cómo se distribuye la $\mathrm{Hb}$ a nivel del mar y a diferentes altitudes. Estos trabajos se hicieron en base a información de trabajadores mineros y con objetivos diferentes al presente estudio. Lamentablemente, en la población infantil, no se ha reportado algún trabajo en este sentido.
Sin embargo, en otros países existen reportes en este grupo poblacional. Así tenemos a Dirren ${ }^{(15)}$, que en una muestra nacional de 469 niños ecuatorianos de 6 a 59 meses y una altitud hasta los 3400 msnm, propuso un factor de corrección en base a una ecuación exponencial. Miao en China también hizo su propia corrección en niñas menores de 1 año y hasta los 4000 msnm, usando el análisis de regresión lineal univariado (16). Ruiz-Arguelles, en México, utilizando ecuaciones algebraicas calcularon los valores de parámetros eritrocíticos, entre ellos la $\mathrm{Hb}$, de sujetos sanos residentes de altura entre 0 y 2670 metros $^{(17)}$. Berger, en Bolivia, en niños de 6 a 108 meses de dos ciudades de Potosí (Atocha 3600 y Santa Bárbara 4800 msnm, respectivamente), con una regresión lineal ${ }^{(18)}$. Amstrong, con la información proporcionada por las Encuestas Demográficas y de Salud Familiar llevadas a cabo en Armenia, India, Kyrgyzstan y Nepal en niños de 6 a 59 meses que vivían hasta los 3212 msnm (19). El CDC con una población de niños comprendidos entre los 24 a 60 meses entre los 1200 y 3000 msnm en zonas montañosas de los Estados Unidos ${ }^{(12)}$.

Todas las mencionadas correcciones presentan algunas diferencias, como las edades del grupo estudiado, altitudes de residencia, diseño metodológico y el análisis de la información. Es por esta razón que su uso se ha limitado a las áreas donde fueron realizados. En ese sentido, el que ha tenido una amplia difusión es el factor de corrección propuesto por el CDC, que es utilizado en los países que sufren la problemática de la anemia (20).

Sin embargo, debe de reconocerse que en la elaboración de la ecuación del CDC no fueron incluidos niños menores de 24 meses y su altitud abarcó solo hasta los $3000 \mathrm{msnm}$. Esto que parece un detalle menor podría tener alguna repercusión, dado que se está utilizando para correcciones de $\mathrm{Hb}$ en niños que viven por encima de los $3000 \mathrm{msnm}$, incluso hasta los 4500 msnm. En vista de lo señalado es necesario proponer un factor de corrección de altitud, donde se contemple cubrir todos los grupos de edad y los niveles de altitud de los Andes. La propuesta presentada tiene como ventaja metodológica el comprender a niños de 
6 a 59 meses, que residen hasta una altura de 4500 msnm y con niños peruanos. Para ello se eligió la información recogida por ENDES 2015 y como único criterio de inclusión se seleccionó los niños no anémicos, con el propósito de eliminar otros factores que pudiesen distorsionar la relación Hb/altitud, como serían inflamaciones, parasitosis o deficiencia de otros micronutrientes.

Con la finalidad de elaborar la ecuación que permitiese tener un factor de corrección se ha utilizado la regresión no lineal tipo exponencial, dado que es la que mejor ajusta o representa tanto a la variable $\mathrm{Hb}$, como también a la variable 'diferencia de la Hb observada menos 110 ' comparada con la variable altura. En razón de estas consideraciones se puede decir que los datos (valores de $\mathrm{Hb}$ ) se distribuyen exponencialmente. Esta misma distribución fue reportada por Hurtado en el Perú (7) y Dirren en el Ecuador (15). Usando el mencionado factor, se logra obtener una prevalencia nacional de $31,8 \%$, menor a lo reportado cuando se utiliza el factor dado por el CDC, que es de $34,3 \%$, con una diferencia de $2,5 \%$.

Adicionalmente, hay que resaltar que en Afganistán, en menores de 5 años encontraron diferencias en las prevalencias usando los niveles de ajuste de la $\mathrm{Hb}$ representados por las tablas de corrección dadas por su Ministerio de Salud con los resultados que se dieron usando la fórmula del CDC ${ }^{(12)}$.

Al desagregar la información por niveles de altitud se observa que, a partir de los 2500 msnm, las diferencias entre nuestra propuesta y la ecuación de la CDC se empiezan a incrementar, haciéndose más ostensible a partir de los 3000 msnm, donde la diferencia alcanza a 7,8\% hasta llegar a 13,9\% por encima de los 4000 msnm. Una posible explicación que se podría dar a esta diferencia es el hecho que el estudio del CDC solo es hasta los 3000 msnm y que las correcciones ofrecidas para mayores altitudes responden a una tendencia estadística. Esto es más evidente cuando la altitud se divide por debajo y por encima de 3000 msnm. Por debajo de los $3000 \mathrm{msnm}$, las prevalencias son prácticamente iguales usando ambos factores, lo que no sucede por encima de los 3000 msnm, donde el factor del CDC identifica un 46,2\% frente a la propuesta de $37 \%$, prácticamente $10 \%$ de diferencia, lo que significa 4 veces más que lo mostrado a nivel nacional.

El panorama se muestra más claro cuando se da la información por departamentos, donde se observa que en la costa y selva sus prevalencias son escasamente diferentes y en algunos iguales, para ambos factores de corrección. Lo contrario sucede con los considerados ubicados en la sierra. Lo que llama la atención son las diferencias que se encuentran, principalmente en Puno y Huancavelica, y esto se debería a que la mayoría de la población estudiada se halla por encima de los 3000 msnm. En ese sentido, los porcentajes de población de niños menores de 5 años que viven por encima de los 3000 msnm son $95 \%$ y $82,3 \%$, respectivamente ${ }^{(21,23)}$.

Analizando el peso que pueden tener esas diferencias de prevalencias con los factores de corrección utilizados, se hace un ejercicio de inferencia poblacional en base a la cantidad de niños de 6 a 59 meses que tiene el país, que llegan a $2,565,709$. En ese sentido, a nivel nacional, las diferencias entre las dos correcciones representan en números absolutos 64143 niños (2,5\%). Con el mismo razonamiento, para Puno son 16535 niños (12,7\%; población total = 130195$)$ y Huancavelica 5856 niños (9,9\%; población total = 59 149) que habrían sido diagnosticados como anémicos sin serlo ${ }^{(21,23)}$.

Si bien no es el objetivo del estudio el referirnos a las gestantes, merece una reflexión en razón de que se usa el mismo criterio diagnóstico para identificar anemia que en los niños preescolares, o sea $\mathrm{Hb}<11 \mathrm{~g} / \mathrm{dL}$. Coincidentemente las mayores prevalencias de anemia en las gestantes la presentan Puno (51\%) y Huancavelica $(53,6 \%)^{(22)}$. En el hipotético caso que la $\mathrm{Hb}$ de la gestante tuviese un comportamiento similar a la del niño, se estaría sobreestimando las prevalencias.

En lo que se refiere a la otra limitante de la corrección del CDC, respecto a los menores de 24 meses, muy posiblemente usaron la misma metodología que para lo mencionado con la altitud y que en ra- zón de lo reportado no tuvieron la misma repercusión en los resultados comparativos, dado que las diferencias estuvieron muy parecidas a las de nivel nacional. Ambos factores muestran que a menor edad hay mayor prevalencia.

Dado que la anemia es considerada como un problema de Salud Pública, se recomienda el uso de la $\mathrm{Hb}$ para identificar la magnitud y localización del problema ${ }^{(8)}$. Sin embargo, no se debe dejar de reconocer que para conocer su etiología existen otros indicadores utilizados en el campo clínico y de difícil aplicación en los estudios poblacionales ${ }^{(24)}$

La limitante del estudio es no haber podido conseguir una información más precisa y desagregada de la metodología usada para la elaboración del factor de corrección propuesto por CDC.

Se concluye que el usar el factor de corrección propuesto en este artículo identifica menores prevalencias de anemia en niños menores de 5 años, que el factor tradicionalmente utilizado, y que esta diferencia se sustenta en las diferencias marcadas que se dan a partir de los 3000 msnm, existiendo la necesidad de conocer en cuánto influye estas variaciones en el diseño de las políticas públicas.

\section{REFERENCIAS BIBLIOGRÁFICAS}

1. Lopez A, Cacoub P, Macdougall I, Peyrin-Biroulet L. Iron deficiency anaemia. Lancet (London, England) [serial on the Internet]. (2016, Feb 27), [cited March 30, 2017];387(10021):907-16.

2. Klaus H, Tam M. Contact in the Andes: bioarchaeology of systemic stress in colonial Mórrope, Peru. Am J Phys Anthropol. [serial on the Internet]. (2009, Mar), [cited March 30, 2017];138(3):356-68. Disponible en: Academic Search Ultimate.

3. Trinkaus E. The Alto Salaverry child: a case of anemia from the Peruvian preceramic. Am J Phys Anthropol. [serial on the Internet]. (1977, Jan), [cited March 30, 2017];46(1):25-8. Disponible en: MEDLINE Complete.

4. Castro de la Mata, R. Hominidae. Homo sapiens sapiens. En: Bonavia D, editor. Precerámico Peruano. Los Gavilanes. Mar, desierto y oasis en la historia del hombre. Lima: Corporación Peruana de Desarrollo S.A. COFIDE, Instituto Arqueológico Alemán; 1982:201-3.

5. Costa-Junqueira MA, Cocilovo JA, Quevedo S. Patologias óseas, traumas y otros atributos en el grupo arcaico de Morro de Arica, norte de Chile. Chungará (Arica) [internet]. 2000 ene [citado 30 mar 2017]; 32(1):79-83. Disponible en: http:// dx.doi.org/10.4067/S0717-73562000000100013 
6. Pamo O. Daniel Vergara Lope y Thomas Holmes Ravenhill: dos figuras olvidadas en la historia de la fisiologia de altura. Rev Med Hered [internet]. 2005 jul [citado 30 mar 2017];16(3):208-17. Disponible en: http://www.scielo.org.pe/scielo. php?script=sci_arttext\&pid=S1018-130X200500 0300009\&lng=es\&nrm=iso\&tlng=es

7. Hurtado A, Merino C, Delgado E. Influence of anoxemia on the hemopoietic activity. Arch Intern Med (Chic) [internet]. 1945 may [citado 31 mar 2017];75(5):284-323. Disponible en: http:// jamanetwork.com/journals/jamainternalmedicine/ article-abstract/551113? resultClick $=3$

8. WHO. Haemoglobin concentrations for the diagnosis of anaemia and assessment of severity. Vitamin and Mineral Nutrition Information System. Geneva, World Health Organization, 2011 (WHO/NMH/NHD/ MNM/11.1) (http://www.who.int/vmnis/indicators/ haemoglobin. pdf, citado [20 mar 2017]).

9. Pajuelo J, Amemiya I. Anemia nutricional en la población infantil del Perú. Rev Méd Peruana. 1992 jun-dic;64(347):50-5.

10. Instituto Nacional de Estadística e Informática. Perú: Encuesta Demográfica y de Salud Familiar -ENDES 2015. Lima Perú: INEI; may 2016. https://www.inei.gob.pe/media/MenuRecursivo/ publicaciones_digitales/Est/Lib1356/index.htm [Acceso 20-03-17].

11. Cartwright GE. Diagnostic Laboratory Hematology, 4th ed. Grune \& Stratton. New York; 1968.

12. CDC criteria for anemia in children and childbearing-aged women. MMWR. Morbidity And Mortality Weekly Report [serial on the Internet]. (1989, June 9), [cited March 30, 2017];38(22):400-4. Disponible en: MEDLINE Complete.
13. Cosio G, Yataco A. Valores de hemoglobina en relación con la altura sobre el nivel del mar. Rev Salud Ocupacional. 1968 jul;13(3-4):5-17.

14. Rodriguez L. Patrones hematológicos en la altura. Rev Salud Ocupacional. 1962 jul;7l(3):42-55.

15. Dirren H, Logman M, Barclay D, Freire W. Altitude correction for hemoglobin. Eur J Clin Nutrition [serial on the Internet]. (1994, Sep), [cited March 30, 2017];48(9):625-32. Disponible en: MEDLINE Complete.

16. Miao G. Normal reference value of hemoglobin of infant girls and altitude in China. Arch Med Res [serial on the Internet]. (2004, Jan), [cited March 30, 2017];35(1):87-90. Disponible en: MEDLINE Complete.

17. Ruiz-Argüelles G, Llorente-Peters A. Predictive equations for normal red cell values at altitudes from sea level to 2,670 meters. Revista De Investigacion Clinica; Organo del Hospital de Enfermedades de la Nutricion [serial on the Internet]. (1981, Apr), [cited March 30, 2017];33(2):191-3. Available from: MEDLINE Complete.

18. Berger J, Aguayo VM, San Miguel JL, Lujan C, Téllez W, Traissac P. Estrategias de control de la anemia ferropénica en niños bolivianos residentes a gran altitud. En: Berger J, San Miguel JL, editores. Anemia por deficiencia de hierro en la región andina: Definición y estrategias de intervención, La Paz: ORSTOM; 1996:227-48.

19. Armstrong A. Anemia in Central-Asia pre-school children: definition, risk factor and evaluation of home fortification intervention [tesis doctoral en internet]. New York: Cornell University; 2009 [citado 20 mar 2017]. 147 p. Disponible en: https://ecommons.cornell.edu/bitstream/
handle/1813/13841/Armstrong\%2c\%20Avril. pdf? sequence $=1$ \&isAllowed $=y$

20. Sullivan K, Mei Z, Grummer-Strawn L, Parvanta I. Haemoglobin adjustments to define anaemia. Tropical Medicine \& International Health: TM \& IH [serial on the Internet]. (2008, Oct), [cited March 30, 2017];13(10):1267-71. Disponible en: MEDLINE Complete.

21. Ministerio de Salud. Oficina General de Tecnologías de la Información. Fuente: Boletines Especiales de Estimaciones y Proyecciones de Población-INEI. Lima 2015.

22. Ministerio de Salud. Instituto Nacional de Salud. Anemia en gestantes del Perú y provincias con comunidades nativas. Informe de la Dirección Ejecutiva de Alimentación y Nutrición (DEVAN) del Centro Nacional de Alimentación y Nutrición (CENAN). Perú: INS/MINSA. Junio 2012. Disponible en: http://www.ins.gob.pe/repositorioaps/0/5/ jer/res_2011/Prevalencia\%20de\%20anemia\%20 en\%20gestantes\%20v\%201_0_1.pdf [acceso el 30-03-2017].

23. Perú, Instituto Nacional de Estadística e Informática. Directorio Nacional de Municipalidades Provinciales, Distritales y de Centros Poblados 2015. Lima: INEI; 2015. Disponible en: https://www.inei. gob.pe/media/MenuRecursivo/publicaciones_digitales/Est/Lib1201/. [acceso el 20-03-17]

24. National Institutes of Health. National Hearth, Lung and Blood Institute. [actualizado: junio 13, 2012; acceso 20 marzo 2017]. ¿Cómo se diagnostica la anemia? [una pantalla]. Disponible en: (www. nhlbi.nih.gov/health-spanish/health-topics/temas/ anemia/diagnosis 